\title{
EMANT: Integration of GiD and Kratos, Open and Flexible Computational Tools
}

\author{
Rubén Otín*, Javier Mora and Eugenio Oñate \\ International Center For Numerical Methods in Engineering (CIMNE) \\ Edifici C1 - Campus Nord UPC \\ c $\backslash$ Gran Capità s/n, 08034 Barcelona (Spain) \\ Tfn: +34 9340173 99, Fax: +34934016517 \\ e-mail: rotin@cimne.upc.edu
}

\section{Introduction}

Modern computational electromagnetism approaches includes coupled problems, hybrid methods, open platforms, flexibility and customized solutions. On one hand, a new trend of general software programmes, like Matlabß or related packages like Femlabß, are growing in popularity thanks to their focus on learning and easy-to-use. On the other hand, industry is demanding specific programs, able to understand their own vocabulary and adapted to the needs of their designers and engineers.

The aim of this paper is to introduce three useful computer tools intended to cope with the approaches mentioned above:

- GiD: [5] is a pre- and post- processor where we can define our geometries, assign materials, mesh and visualize the results of our computational analysis, with powerful capabilities to design graphic interfaces according to users' preferences.

- Kratos: [1] is an open source $\mathrm{C}++$ object-oriented library designed for the development of multiphysics analysis software.

- EMANT: [6] is a finite-element software package for solving Maxwell's equations. It can be seen as an example of integration between Kratos and GiD for electromagnetic analysis.

The key feature of this tools is that they can be used to develop customized software packages: GiD can be set for being used with all types of numerical simulation programs and Kratos is open source, i.e., the main code and program structure is fully accessible, and you are allowed to use and distribute the existing code without any restriction.

\section{GiD: pre- and post- processor}

GiD is a universal, adaptive and user-friendly graphical interface for geometrical modeling, data input and visualization of results. It can be customized for being used with all types of numerical simulation programs (see fig.1). 

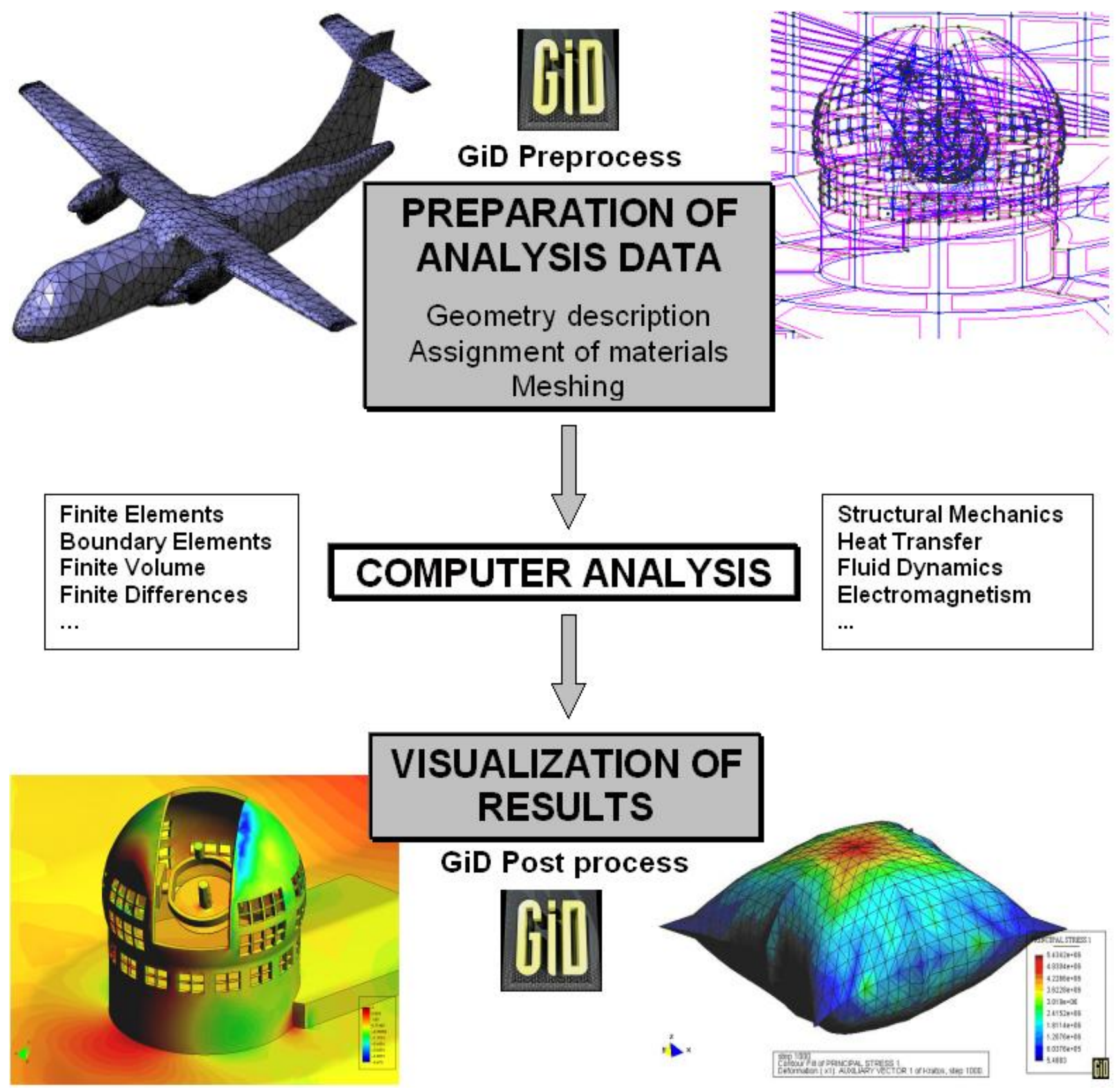

Figure 1: GiD diagram 
Typical problems that can be successfully tackled with GiD include most situations in solid and structural mechanics, fluid dynamics, electromagnetism, heat transfer, geomechanics, etc. using finite element, finite volume, boundary element, finite difference or point based (meshless) numerical procedures (visit http://gid.cimne.upc.es/2004/papers.subst to see some advanced applications of GiD, including some applications to electromagnetism).

GiD can generate all the information (structured and unstructured meshes, boundary and loading conditions, material types, visualization of results, etc.) required for the numerical analysis of any problem in science and engineering.

GiD is extremely easy to adapt to any simulation code. In fact, GiD can be defined by the user to read and write data in an unlimited number of formats. GiD's input and output formats can be customized and made compatible with any existing in-house software. The different menus for data input and results visualization can be tailored to the specific needs and desires of the user.

GiD has been designed to be used in universities, research centers and companies to develop and apply different numerical simulation programs. However, in spite of its advantageous features, GiD has some limitations for electromagnetic problems, mainly due to the unavailability of non-standard (H(curl) and $\mathrm{H}(\mathrm{div})$, low and higher orders) finite elements.

\section{Kratos: an object-oriented environment for the development of multi-physics analysis software.}

Kratos is a $\mathrm{C}++$ open source framework with object-oriented structure aimed to provide computer tools to finite element developers. It consists in a set of classes and methods that allow programmers to handle with multi-physic, adaptive meshing and optimization problems.

Kratos should help to built a numerical application in $\mathrm{C}++$ from the easiest formulation (conduction problem) to the most complex ones (optimization techniques). It is possible to work with Kratos as developer and also as user of the already existing codes, employing it as calculation engine.

Kratos is open source, i.e., the main code and program structure is available under GNU Lesser General Public License and the code can be open or close depending on the developers preferences.

The major drawback of Kratos current version for computational electromagnetism is that does not work with complex numbers and that it only deals with Langrangian elements, but, in next versions, both problems are planned to be solved. Also, Kratos will include the possibility of using phyton to interact with the database on run time, in order to extend their set of functions to be adapted to the user requirements.

\section{EMANT: GiD and Kratos applied to electromagnetic problems}

EMANT is a finite-element code for solving Maxwell's equations. It has been developed with Kratos and uses GiD as pre- and post- processor. Its range of application goes from static to high frequency 
electromagnetism. At present time, EMANT can solve 2D and 3D linear problems in frequency domain. A quasi-static 2D module in time domain for eddy current typical problems has been also developed.

A full 2D electromagnetic module (from static to high frequency problems) is available in [6], with some examples of use and an user manual. 3D modules are under development, but available for some general applications. The formulation used is detailed in the documentation section of [6] and is mainly based on [4] for the 2D module and on the papers [3,2] for the 3D module.

In a near future, taking advantage of Kratos inherent modularity, EMANT is intended to cope with multi-physics coupled problems and hybrid methods like BEM-FEM or GTD-FEM.

The main goal of the current version is help students, scientist and engineers to calculate electromagnetic fields in a wide variety of practical situations, helping also to reach a deeper knowledge of electromagnetic phenomena by showing fields behavior visually.

\section{Conclusion}

The advantages and drawbacks of GiD, Kratos and EMANT has been presented. The main feature of these computer tools is that they can be used to craft customized numerical simulation programs: GiD for geometrical modeling, data input and visualization of results. Kratos as starting point for a $\mathrm{C}++$ finite element code, and finally, EMANT, can be seen as an example of how to blend Kratos and GiD to analyze electromagnetic problems.

\section{References}

[1] KRATOS: An Object-Oriented Environment for Development of Multi-Physics Analysis Software. http://www.cimne.com/kratos. International Center for Numerical Methods in Engineering (CIMNE).

[2] C. Hazard and M. Lenoir. On the solution of the time-harmonic scattering problems for Maxwell's equations. SIAM Journal on Mathematical Analysis, 27:1597-1630, 1996.

[3] Bo-Nan Jiang, Jie Wu, and L.A. Povinelli. The origin of spurious solutions in computational electromagnetics. Journal of Computational Physics, 125:104-123, 1996.

[4] J. Jin. The Finite Element Method in Electromagnetics. John Wiley \& Sons, 2002.

[5] GiD:Personal Pre and Post Processor. http://www.gidhome.com. International Center for Numerical Methods in Engineering (CIMNE).

[6] EMANT-Kratos: Electromagnetic Analysis with Numerical Tools using a Multi-Physics ObjectOriented Environment Software. http://www.cimne.com/kratos/emant. International Center for $\mathrm{Nu}-$ merical Methods in Engineering (CIMNE). 